\title{
Correlation of VITEK -2 and conventional tests in identification of Non Fermenting Gram Negative Bacilli in a Tertiary Care Hospital
}

\author{
Shweta Sharma ${ }^{1}$, R K Agarwal,", Barnali Kakati ${ }^{3}$ \\ ${ }^{1}$ Junior Resident, ${ }^{2}$ Professor \& Head, ${ }^{3}$ Professor, Dept. of Microbiology, Himalayan Institute of Medical Sciences, Swami Rama \\ Himalayan University, Dehradun, Uttarakhand, India
}

Corresponding Author:

Email: rka1951@gmail.com

\begin{abstract}
Introduction: The non-fermentative Gram negative bacilli (NFGNB) are a group of aerobic, non-spore forming, Gram negative bacilli that either do not utilize carbohydrates as a source of energy or degrade them through metabolic pathways other than the fermentation.

Material and Methods: The study was undertaken to identify various non- fermenters isolated from patients in our hospital, a Tertiary Care Center at Uttarakhand. 252 (14.15\%) non-fermenters were isolated from 1780 clinical specimens on MacConkey and blood agar plates incubated at $35^{\circ} \mathrm{C}$ for $24-48$ hours. Any growth that was identified as non-fermenters by VITEK-2 was subjected to identification by additional conventional tests.

Results and Conclusion: Patients mostly belonged to age group of 51 to 70 years and were commonly male. Sputum \& ET secretion were the commonest samples. Psuedomonas aeruginosa was the commonest isolate followed by Acinetobacter calcoacetecus baumanii complex. Out of total 252 isolates, 221(87.69\%) could be accurately identified upto species level by conventional tests. The conventional tests could identify $243(96.42 \%)$ isolates upto genus level. Majority of them showed multidrug resistance. The most effective antibiotics were Tigecycline and Polymyxin B/ Colistin.
\end{abstract}

Keywords: Non- fermenters, VITEK -2, Additional conventional biochemical tests.

\section{Introduction}

Non-fermenting Gram negative bacilli (NFGNB) are a taxonomically diverse group of aerobic, non-sporing bacilli that either do not utilize glucose as a source of energy or utilize it oxidatively rather than fermentatively. ${ }^{1}$

Although generally considered as contaminants, their pathogenic potential have been proved beyond doubt by their frequent isolation repeatedly from clinical material and their association with disease ${ }^{2}$

NFGNB have emerged as important healthcare-associated pathogens. They have been incriminated in infections such as septicemia, meningitis, pneumonia, urinary tract infections (UTI) ${ }^{3}$ and surgical site infections (SSI) . ${ }^{4}$

Non-fermenters may differ in their pathogenic potential and transmissibility, and many are multidrug resistant. ${ }^{5}$ They have a low degree of virulence ${ }^{1}$ and most often cause nosocomial infections ${ }^{6}$ in patients who are debilitated or immunocompromised. ${ }^{7}$

The group includes diverse genera like Pseudomonas, Acinetobacter, Stenotrophomonas, Burkholderia, Flavobacterium, Chryseobacterium, Weeksella, Spingobacterium, Moraxella, Psychrobacter, Bordetella, Ralstonia etc. ${ }^{1}$ They share the common phenotypic feature of failing to acidify the butt of Triple sugar iron agar or Kligler iron agar.

As NFGNB are known to account for about $15 \%$ of all bacterial isolates from a clinical laboratory, ${ }^{8}$ the rapid and accurate identification is necessary for appropriate management of the patient.
Hence,the present study was undertaken to identify and speciate NFGNB isolated from various clinical specimens from our hospital and to evaluate the role of conventional tests for their identification viz a viz automated system like VITEK-2.

\section{Materials and Methods}

The prospective study was conducted in the Department of Microbiology, Himalayan Institute of Medical Sciences (HIMS), Dehradun, India, after taking approval from Research and Ethical Committee of our institute during the period of January 2016 to December 2016.

252 non-fermenters were isolated from 1780 clinical specimens like sputum, blood, pus, swabs of ulcer, pleural fluids, ascitic fluid, tracheal secretions and urine. The specimens were cultured on MacConkey and blood agar at $35^{\circ} \mathrm{C}$ for $24-48$ hours of incubation. Any growth that was identified as non-fermenters by VITEK-2 was subjected to identification by additional conventional tests.

\section{Isolate Speciation / Identification}

In the diagnostic clinical microbiology laboratory, identification of non-fermenters relies mainly on phenotypic characteristics. NFGNB are presumptively identified by colonial morphology, Gram stain, oxidase activity and pigment production. ${ }^{6}$ Routinely they are identified only in few laboratories in India as they are slow growing and require special culture media and biochemical tests for their identification. 
In our laboratory we are using VITEK-2 for identification. Any growth that was identified as nonfermenters by automated identification system VITEK2 (bioMe'rieux) was subjected to identification by additional conventional tests like Oxidase test, Motility by Hanging drop method, Nitrate reduction test, Indole test, Tyrosine-Arylamidase test, Urea hydrolysis test, Citrate utilization test, Malonate utilization test,
Phosphatase, L-Malate assimilation test, $\mathrm{H}_{2} \mathrm{~S}$ Production in TSI medium, Ornithiine and Lysine Decarboxylases, and Arginine Dehydrolase, Formation of acid from D-Celliobiose, Beta-Galactosidase, DGlucose, D-Maltose, D-Mannitol, D-Mannose and Sucrose in HL media (Table 1).

Table 1: Identification of NFGNB with conventional laboratory methods

\begin{tabular}{|c|c|c|c|c|c|c|c|c|c|c|c|c|c|}
\hline Biochemicals & 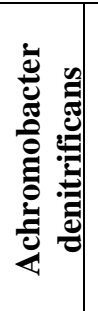 & 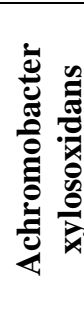 & 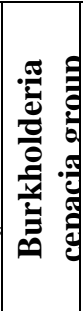 & 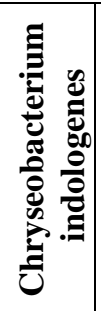 & 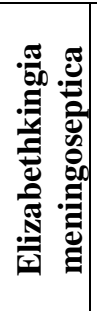 & 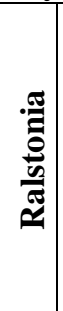 & 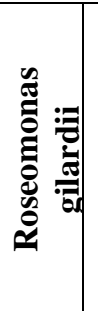 & 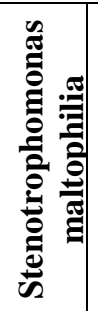 & 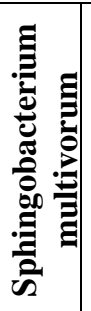 & 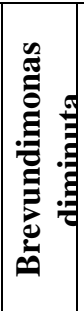 & 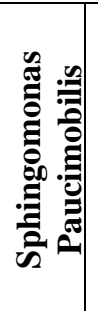 & 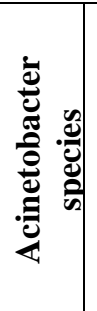 & 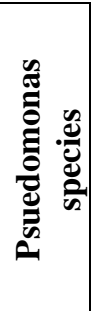 \\
\hline $\begin{array}{l}\text { Growth on Mac } \\
\text { Conkey }\end{array}$ & + & + & + & + & + & + & + & + & V & V & V & + & + \\
\hline Oxidase test & + & + & $\mathrm{V}$ & + & $\mathrm{V}$ & + & + & + & $\mathrm{V}$ & $\mathrm{V}$ & - & - & + \\
\hline $\begin{array}{l}\text { Motility by Hanging } \\
\text { drop method }\end{array}$ & + & + & + & - & + & + & + & + & - & - & V & - & + \\
\hline Nitrate reduction test & - & - & + & - & $\mathrm{V}$ & $\mathrm{V}$ & - & $\mathrm{V}$ & $\mathrm{V}$ & - & + & - & + \\
\hline Indole test & - & - & - & + & + & - & - & - & 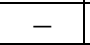 & - & 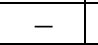 & - & 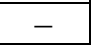 \\
\hline \multicolumn{14}{|l|}{$\begin{array}{l}\text { Formation of acid in } \\
\text { HL media in :- }\end{array}$} \\
\hline D-Celliobiose & - & - & $\mathrm{V}$ & + & - & - & - & - & $\mathrm{V}$ & - & $\mathrm{V}$ & + & - \\
\hline Beta-Galactosidase & - & - & $\mathrm{V}$ & - & $\mathrm{V}$ & - & - & - & + & - & $\mathrm{V}$ & - & - \\
\hline D-Glucose & $\mathrm{V}$ & + & + & $\begin{array}{lllll}- & \end{array}$ & 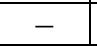 & + & - & $\mathrm{V}$ & + & 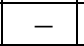 & $\mathrm{V}$ & + & + \\
\hline D-Maltose & - & - & $\mathrm{V}$ & $\mathrm{V}$ & $\mathrm{V}$ & $\mathrm{V}$ & - & $\mathrm{V}$ & + & - & $\mathrm{V}$ & - & - \\
\hline D-Mannitol & - & - & $\mathrm{V}$ & - & - & + & - & - & $\mathrm{V}$ & - & $\mathrm{V}$ & - & - \\
\hline D-Mannose & - & - & $\mathrm{V}$ & - & $\mathrm{V}$ & $\mathrm{V}$ & - & $\mathrm{V}$ & + & - & $\mathrm{V}$ & + & $\mathrm{V}$ \\
\hline Sucrose & - & - & + & - & - & - & - & - & $\mathrm{V}$ & - & $\mathrm{V}$ & - & - \\
\hline Tyrosine-Arylamidase & $\mathrm{V}$ & - & - & + & + & + & + & - & $\mathrm{V}$ & + & $\mathrm{V}$ & + & $\mathrm{V}$ \\
\hline Urea hydrolysis test & & - & - & - & - & + & + & - & $\mathrm{V}$ & - & - & - & - \\
\hline Citrate utilization test & + & + & + & $\mathrm{V}$ & $\mathrm{V}$ & + & + & $\mathrm{V}$ & - & - & $\mathrm{V}$ & + & + \\
\hline $\begin{array}{l}\text { Malonate utilization } \\
\text { test }\end{array}$ & - & - & $\mathrm{V}$ & - & - & + & - & V & - & - & - & + & $\mathrm{V}$ \\
\hline Phosphatase & - & - & $\mathrm{V}$ & + & + & - & - & + & $\mathrm{V}$ & + & $\mathrm{V}$ & - & - \\
\hline L-Malate assimilation & $\mathrm{V}$ & - & $\mathrm{V}$ & - & - & - & - & - & - & - & - & - & $\mathrm{V}$ \\
\hline $\begin{array}{l}\text { H2S Production in } \\
\text { TSI medium }\end{array}$ & - & - & - & - & - & - & - & - & - & - & - & - & - \\
\hline $\begin{array}{l}\text { Ornithiine } \\
\text { Decarboxylase }\end{array}$ & - & - & - & - & - & - & - & - & - & - & - & - & - \\
\hline Lysine Decarboxylase & - & $\begin{array}{llll}- & & & \end{array}$ & - & - & - & - & - & - & - & - & - & - & $\begin{array}{llll}- & \\
\end{array}$ \\
\hline Arginine Dehydrolase & - & - & - & $\mathrm{V}$ & - & - & - & - & - & - & - & - & $\mathrm{V}$ \\
\hline $\begin{array}{l}\text { Pigments in } \\
\text { king's A \& B }\end{array}$ & - & - & - & - & - & - & - & - & - & - & - & - & + \\
\hline
\end{tabular}

$\mathrm{V}$ means variable.

\section{Results}

Two hundred fifty two non-fermenters were isolated from 1780 clinical specimens which were submitted to microbiology laboratory. The isolation rate of NFGNB was found to be $14.15 \%$.
The most common age group yielding NFGNB was found to be 51-70 years.

Males more commonly yielded $\operatorname{NFGNB}(61 \%)$ as compared to females (39\%).The commonest specimen yielding the growth of NFGNB were sputum/ET secretions $(35.32 \%)$ followed by pus (20.63\%) . By 
employing a few simple additional tests we could

level and 221 (87.69\%) upto species level (Table 2). correctly identify $243(96.42 \%)$ isolates upto genus

Table 2: Correlation of VITEK-2 and conventional tests in identification of NFGNB

\begin{tabular}{|c|c|c|c|c|c|c|c|}
\hline $\begin{array}{c}\text { VITEK-2 } \\
\text { system } \\
\text { identitification }\end{array}$ & $\begin{array}{c}\text { Number of } \\
\text { isolates } \\
\text { and } \\
\text { Percentage }\end{array}$ & $\begin{array}{c}\text { Identification by } \\
\text { routine } \\
\text { laboratory } \\
\text { methods } \\
\text { upto species level }\end{array}$ & $\begin{array}{l}\text { Number of } \\
\text { isolates } \\
\text { and } \\
\text { percentage }\end{array}$ & 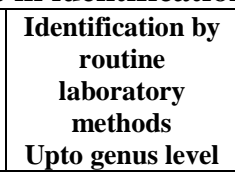 & $\begin{array}{l}\text { Number of } \\
\text { isolates and } \\
\text { percentage }\end{array}$ & $\begin{array}{l}\text { Not identified } \\
\text { By routine } \\
\text { laboratory } \\
\text { methods }\end{array}$ & $\begin{array}{c}\text { Number of } \\
\text { isolates } \\
\text { and } \\
\text { percentage }\end{array}$ \\
\hline $\begin{array}{c}\text { Acinetobacter } \\
\text { baumanii complex }\end{array}$ & $\begin{array}{c}65 \\
(100 \%) \\
\end{array}$ & $\begin{array}{c}\text { Acinetobacter } \\
\text { baumanii complex }\end{array}$ & $\begin{array}{c}61 \\
(93.8 \%) \\
\end{array}$ & $\begin{array}{c}\text { Acinetobacter } \\
\text { spp }\end{array}$ & $\begin{array}{c}3 \\
(4.61 \%) \\
\end{array}$ & $\begin{array}{c}\text { Acinetobacter } \\
\text { baumanii complex }\end{array}$ & $\begin{array}{c}1 \\
(1.5 \%)\end{array}$ \\
\hline $\begin{array}{l}\text { Burkholderia } \\
\text { cepacia group }\end{array}$ & $\begin{array}{c}18 \\
(100 \%)\end{array}$ & $\begin{array}{l}\text { Burkholderia } \\
\text { cepacia group }\end{array}$ & $\begin{array}{c}16 \\
(88.88 \%)\end{array}$ & Burkholderia spp & $\begin{array}{c}1 \\
(5.55 \%)\end{array}$ & $\begin{array}{l}\text { Burkholderia } \\
\text { cepacia group }\end{array}$ & $\begin{array}{c}1 \\
(5.55 \%)\end{array}$ \\
\hline $\begin{array}{l}\text { Elizabethkingia } \\
\text { meningoseptica }\end{array}$ & $\begin{array}{c}11 \\
(100 \%)\end{array}$ & $\begin{array}{l}\text { Elizabethkingia } \\
\text { meningoseptica }\end{array}$ & $\begin{array}{c}10 \\
(90.90 \%)\end{array}$ & $\begin{array}{l}\text { Elizabethkingia } \\
\text { spp }\end{array}$ & $\begin{array}{c}1 \\
(9.09 \%)\end{array}$ & $\begin{array}{l}\text { Elizabethkingia } \\
\text { meningoseptica }\end{array}$ & $\begin{array}{c}0 \\
(0.00 \%)\end{array}$ \\
\hline $\begin{array}{l}\text { Psuedomonas } \\
\text { aeruginosa }\end{array}$ & $\begin{array}{c}93 \\
(100 \%)\end{array}$ & $\begin{array}{l}\text { Psuedomonas } \\
\text { aeruginosa }\end{array}$ & $\begin{array}{c}89 \\
(95.69 \%)\end{array}$ & Psuedomonas spp & $\begin{array}{c}3 \\
(3.22 \%)\end{array}$ & $\begin{array}{c}\text { Psuedomonas } \\
\text { Aeruginosa }\end{array}$ & $\begin{array}{c}0 \\
(0.00 \%)\end{array}$ \\
\hline $\begin{array}{l}\text { Sphingomonas } \\
\text { paucimobilis }\end{array}$ & $\begin{array}{c}18 \\
(100 \%)\end{array}$ & $\begin{array}{l}\text { Sphingomonas } \\
\text { paucimobilis }\end{array}$ & $\begin{array}{c}14 \\
(77.77 \%)\end{array}$ & Sphingomonas spp & $\begin{array}{c}2 \\
(11.11 \%)\end{array}$ & $\begin{array}{l}\text { Sphingomonas } \\
\text { paucimobilis }\end{array}$ & $\begin{array}{c}2 \\
(11.11 \%)\end{array}$ \\
\hline $\begin{array}{l}\text { Stenotrophomonas } \\
\text { maltophilia }\end{array}$ & $\begin{array}{c}11 \\
(100 \%)\end{array}$ & $\begin{array}{c}\text { Stenotrophomonas } \\
\text { maltophilia }\end{array}$ & $\begin{array}{c}8 \\
(72.72 \%)\end{array}$ & $\begin{array}{l}\text { Stenotrophomonas } \\
\text { spp }\end{array}$ & $\begin{array}{c}3 \\
(27.27 \%)\end{array}$ & $\begin{array}{c}\text { Stenotrophomonas } \\
\text { maltophilia }\end{array}$ & $\begin{array}{c}0 \\
(0.00 \%)\end{array}$ \\
\hline
\end{tabular}

P.aeruginosa (36.90\%) was found to be most common isolate in our study followed by A.baumanii $(26.19 \%)$.

We were able to identify some rare NFGNB like Achromobacter denitrificans, Burkholderia cepacia group, Chryseobacterium indologenes, Elizabethkingia Meningoseptica, Moraxella group, Sphingomonas paucimobilis, Stenotrophomonas maltophilia and Roseomonas gilardii.

NFGNB displays a wide and variable spectrum of antibiotic resistance. There was no antibiotic to which all strains were susceptible, but they were mostly sensitive to Tigecycline and Polymyxin B/ Colistin. Most of the NFs isolated were resistant to Penicillin group of drugs, Gentamicin and Ciprofloxacin.

\section{Discussion}

Aerobic Non-Fermenting Gram Negative Bacilli (NFGNB) usually considered as contaminants are emerging as important nosocomial pathogens. Their frequent isolation repeatedly from clinical material and their association with disease supports this view. ${ }^{2}$ The NFGNB have been reported by various workers with a wide frequency ranging from $3.58 \%$ to $15 \% .{ }^{8}$ In the present study also the isolation rate of NFGNB was found to be $14.15 \%$.

The lower isolation rates of NFGNB may be due to the fact that they were often considered as contaminants and ignored by clinicians and laboratory personnel. Moreover, most of the laboratories do not use automated systems for identification or perform additional relatively uncommon tests for their identification.

All the isolate were identified by VITEK-2 system in addition to routine method. The purpose of our study was to evaluate identification and management of the NFGNB by conventional methods. Since, automated systems are not available in small and medium sized laboratory, by employing a few additional tests, thus eliminating the need of costly VITEK-2.

By employing a few simple additional tests we could correctly identify $96.42 \%$ isolates upto genus level and $87.69 \%$ upto species level.

In our study P.aeruginosa (36.90\%) was found to be most common isolate followed by A.baumanii (37.07\%).

Majority of the NFGNB showed multidrug resistance pattern in their antibiotic susceptibility testing. There was no antibiotic to which all strains were susceptible, but they were mostly sensitive to Tigecycline and Polymyxin B/ Colistin

The reason, for these variation in results of NF's in different studies may be due to existing local difference in emergence of drug resistant bacteria and can also be explained by the fact that NF's are found in nature as inhabitants of soil and water.

NFGNB have been proved beyond doubt to be pathogens. Since they differ in their pathogenic potential, transmissibility and antibiotic susceptibility, their accurate identification is important for appropriate patient management.

\section{Limitation}

The limitation of our study was that even by additional tests some of the NFGNB's could not be identified upto the species level as they required some other specific additional tests which are done by VITEK-2 system like Pyrrolydonyl Ayrlamidase, YGlutanyl Transferase, Glucosidase, 1-Proline Arylamidase, Lipase, L-Lactate alkalinisation, Succinate alkinasation and Galactosidase. By employing these tests also, one would be able to identify almost each and every NFGNB. 


\section{Conclusion}

Majority $(96.42 \%)$ of the NFGNB can be identified accurately upto genus level by employing a few additional conventional tests, thus eliminating the need of costly automated systems.

\section{References}

1. Koneman E, Allen S, Janda W, SchreckenbergerP,Winn W. Colour Atlas and textbook of Diagnostic Microbiology. 6th Edition. Philadelphia: Lippincott Raven Publishers. 2006; p. 305-91.

2. Aliaga L, Mediavilla JD, Cobo F. A clinical index predicting mortality with Pseudomonas aeruginosa bacteremia. J Med Microbiol. 2000; 51(7): 615-9.

3. Bergogne, Berezin E, Towner KJ. Acinetobacter spp. as Nosocomial Pathogens: Microbiological, Clinical and Epidemiological Features. ClinMicrobiol Rev. 1996;9(2):148-65.

4. Vijaya D, Kamala, BavaniS, Veena M. Prevalence of nonfermenters in clinical specimens. Indian J Med Sci. 2004;54:87-91.

5. Schreckenberger PC, Daneshvar MI, WeyantRS, Hollis DG. Acinetobacter, Achromobacter, Chryseobacterium, Moraxella, and other nonfermentative Gram-negative rods. In: Murray PR, Baron EJ, Jorgensen JH, Landry ML, Pfaller MA, editors. Manual of Clinical Microbiology. 9th edn. Washington (DC): American Society for Microbiology. 2009;p.770-802.

6. Nagoba BS, Deshmukh SR, Ulka G Gude. Invitro susceptibility of Pseudomonas aeruginosa to different antibiotics. Indian J Med Microbiol. 1997;15:185-6.

7. Gardner P, Griffin WB, Swartz MN, Kunz LJ. Nonfermentative gram-negative bacilli of nosocomial interest. Am J Med. 1970;48(6):735-49.

8. Mehta M, Punia JN, Joshi RM. Antibiotic resistance in Pseudomonas aeruginosa strains isolated from various clinical specimens- a retrospective study. Indian J Med Microbiol. 2001;19(4):232.

9. Benachinmardi KK, Padmavathy M, Malini J, Naveneeth BV. Prevalence of non-fermenting Gram-negative bacilli and their in vitro susceptibility pattern at a tertiary care teaching hospital. J Sci Soc. 2014;41:162-6. 